\title{
BMJ Open Does use of point-of-care testing improve cost-effectiveness of the NHS Health Check programme in the primary care setting? A cost- minimisation analysis
}

Austen El-Osta, ${ }^{1}$ Maria Woringer, ${ }^{1}$ Elena Pizzo, ${ }^{2}$ Talitha Verhoef, ${ }^{2}$ Claire Dickie, ${ }^{1}$ Melody Z Ni, ${ }^{3}$ Jeremy R Huddy, ${ }^{3}$ Michael Soljak, ${ }^{1}$ George B Hanna, ${ }^{3}$ Azeem Majeed $^{1}$

To cite: El-0sta A, Woringer M, Pizzo E, et al. Does use of point-of-care testing improve cost-effectiveness of the NHS Health Check programme in the primary care setting? A cost-minimisation analysis. BMJ Open 2017;7:e015494. doi:10.1136/ bmjopen-2016-015494

- Prepublication history for this paper is available online. To view these files, please visit the journal online (http://dx.doi. org/10.1136/bmjopen-2016015494).

Received 19 December 2016 Revised 18 May 2017

Accepted 19 May 2017

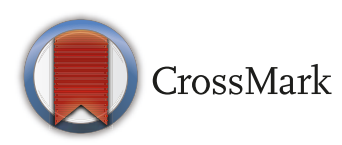

${ }^{1}$ Department of Primary Care and Public Health, Imperial College London, London W6 8RP, UK

${ }^{2}$ Department of Applied Health Research, University College London, London WC1E 7HB, UK ${ }^{3}$ Department of Surgery and Cancer, NIHR DEC London, Imperial College London, London W2 JNY, UK

Correspondence to

Dr Austen El-0sta;

a.el-osta@imperial.ac.uk

\section{ABSTRACT}

Objective To determine if use of point of care testing (POCT) is less costly than laboratory testing to the National Health Service (NHS) in delivering the NHS Health Check (NHSHC) programme in the primary care setting.

Design Observational study and theoretical mathematical model with microcosting approach.

Setting We collected data on NHSHC delivered at nine general practices (seven using POCT; two not using POCT). Participants We recruited nine general practices offering NHSHC and a pathology services laboratory in the same area.

Methods We conducted mathematical modelling with permutations in the following fields: provider type (healthcare assistant or nurse), type of test performed (total cholesterol with either lab fasting glucose or $\mathrm{HbA1c}$ ), cost of consumables and variable uptake rates, including rate of non-response to invite letter and rate of missed [did not attend (DNA)] appointments. We calculated total expected cost (TEC) per 100 invites, number of NHSHC conducted per 100 invites and costs for completed NHSHC for laboratory and POCT-based pathways. A univariate and probabilistic sensitivity analysis was conducted to account for uncertainty in the input parameters.

Main outcome measures We collected data on cost, volume and type of pathology services performed at seven general practices using POCT and a pathology services laboratory. We collected data on response to the NHSHC invitation letter and DNA rates from two general practices.

Results TEC of using POCT to deliver a routine NHSHC is lower than the laboratory-led pathway with savings of $£ 29$ per 100 invited patients up the point of cardiovascular disease risk score presentation. Use of POCT can deliver NHSHC in one sitting, whereas the laboratory pathway offers patients several opportunities to DNA appointment.

Conclusions TEC of using POCT to deliver an NHSHC in the primary care setting is lower than the laboratory-led pathway. Using POCT minimises DNA rates associated with laboratory testing and enables completion of NHSHC in one sitting.
Strengths and limitations of this study

- This is the first study describing cost savings to the NHS in delivering an NHS Health Check using POCT in the primary care setting.

- The mathematical model was based on observed response rates to NHS Health Check invite letters and missed appointments in POCT pathway and costs of phlebotomy and POC testing.

- This study only looked at the cost-saving use of POCT up to the point of cardiovascular disease risk score presentation and did not include full health economic modelling to determine costeffectiveness.

- Traditional laboratory-led pathway offers up to three times more opportunities for a patient to miss an NHS Health Check appointment or to exit the care pathway compared with POCT pathway. This enables general practices to act as a 'one stop shop' to patients wishing to undertake their Health Check in one sitting.

\section{INTRODUCTION}

Cardiovascular disease (CVD) is a major cause of disability and premature mortality worldwide accounting for around one-third of all deaths annually in England. ${ }^{12}$ The National Health Service (NHS) Health Check programme is a national CVD primary prevention programme in England launched by the Department of Health in April 2009. ${ }^{3}$ The programme aims to prevent heart disease, stroke, diabetes and kidney disease while reducing health inequalities. ${ }^{4}$ People aged 40-74 years and free of vascular disease diagnosis are eligible for an NHS Health Check. The programme uses information from the Health Check to calculate a CVD risk score. People at increased risk of CVD 
are offered statins, advice and behaviour change support for lifestyle-related factors, including smoking, physical activity, alcohol consumption and diet. The programme has a potential to prevent 1600 heart attacks and strokes, 650 premature deaths and over 4000 new cases of diabetes each year. ${ }^{5}$ The projected programme cost is $£ 180-£ 243$ million/year at full implementation with estimated cost per quality adjusted life year (QALY) being $£ 3000^{6}$.

The programme is currently delivered in general practice (GP) using both laboratory and point-of-care testing (POCT) equipment to assess blood sugar (glucose or HbA1c) and total cholesterol levels. GPs relying on laboratories first schedule phlebotomy tests and afterwards conduct the full NHS Health Check using glucose and cholesterol readings to calculate and communicate CVD risk. This approach of delivering the programme requires the patient to come back for another visit following laboratory testing to complete the NHS Health Check and delays the communication of GVD risk to patients. The NHS Health Check programme delivered using POCT equipment obtains immediate total cholesterol and glucose/HbAlc results using blood samples taken from 'finger pricking' patients. The use of POCT facilitates communication of CVD risk score to patients in a single visit.

According to the results of a national study, coverage defined as the proportion of total eligible population who attended an NHS Health Check was low (21.4\%) in the first 4 years after programme implementation. ${ }^{7}$ Further, only one-third of attendees at high risk were prescribed a statin after the NHS Health Check. ${ }^{8}$ Another national study found coverage to be low in the first 4 years but steadily rising from $5.8 \%$ in 2010 to $30.1 \%$ in 2012 . Of those found to be at high CVD risk ( $\geq 20 \%, 10$-year risk) when attending an NHS Health Check, only one-fifth were newly prescribed statins. Low NHS Health Check programme coverage and statin prescribing may be exacerbated by higher rates of non-attendance (did not attend, DNA) associated with phlebotomy testing. ${ }^{9}$ Although the use of POCT may lead to better coverage and higher rates of statin prescribing, POCT is not uniformly used.

The primary aim of this analysis was to investigate a range of NHS Health Check screening scenarios to determine if routine use of POCT is less costly than laboratory testing to the NHS.

\section{METHODS}

\section{Data collection from contracted laboratory service}

Data were sought from a local pathology services laboratory that provides services to GPs in Northwest London to illustrate various elements and costs. Data were collected on number and breakdown of various tests requested from GPs, internal and external costs of tests, transport/ courier costs (2013-2014), cost of consumables (20132014) and other infrastructure costs (2013-2014).

\section{GP recruitment}

We collected data on NHS Health Check delivered at nine GPs ( $\mathrm{n}=7$ using POCT; $\mathrm{n}=2$ not using POCT) between September 2013 and August 2014. Nine GPs (eight using TPP SystmOne and one using EMIS Web) with a combined list size of 71500 patients (median=7009) were recruited from four Clinical Commissioning Group localities in Northwest London. Two practices offered NHS Health Check in the traditional 'laboratory model' (LAB) whereby blood samples are processed overnight in a pathology services laboratory either following delivery of the sample to the pathology laboratory (LAB-1 pathway) or following phlebotomy in secondary care (LAB-2 pathway). Seven practices used the Alere Cholestech LDX Analyzer POC device to measure total cholesterol and glucose levels following a pin prick blood sample to inform results of the NHS Health Check. Practices using POCT for this purpose were each loaned a device free of charge by the Public Health England Local Area Team (PHE LAT) in a bid to increase uptake of NHS Health Check in their locality. The cost range of the POC device used in the analysis was $£ 950-£ 1500$. Data on practice list size, number of patients eligible for NHS Health Check, uptake rate, practice workforce configuration, patient flow and other relevant parameters were recorded for each practice. The lineage, nature and time required for each procedure undertaken to enable a Health Check leading up to CVD risk score presentation was recorded, described and timed on three separate occasions resulting in a process map illustrating typical patient journey in either Health Checks delivery model. Aggregate, quantitative and non-patient-identifiable (ie, pseudonymised) data were collected from each participating practice.

\section{UNDERSTANDING PATIENT FLOW AND PROCESS TIMES TO ADMINISTER AN NHS HEALTH CHECK}

GP staff were asked to describe how patients are identified and invited and what processes were involved in administering the NHS Health Check in each respective practice. Three mock NHS Health Check were conducted for each pathway to enable measurement of each relevant procedure. The total average time required to deliver each component of the Health Check in the primary care setting was measured and expressed in minutes and seconds, resulting in a total average time to conduct a Health Check in either POCT or LAB pathway.

\section{CONFIRMING COMPLIANCE OF GP TO PHE CARE QUALITY CRITERIA FOR USING POCT}

All seven GPs using POCT were assessed against the four PHE Care Quality Criteria for using POCT ${ }^{10}$ to confirm that (1) relevant staff have been trained, (2) that there is a named POCT coordinator at each practice, (3) that an appropriate internal quality control process is in place and (4) that each POCT location is registered. 
RATE OF NON-RESPONSE TO INITIAL INVITE AND RATE OF MISSED FOLLOW-UP NHS HEALTH CHECK APPOINTMENTS

Data on the number of patients who do not respond (DNR) to NHS Health Check invite letters and the number of patients who ultimately DNA initial or follow-up appointment are not routinely collected by GPs or PHE LAT. To address this gap in knowledge, four GPs (two using POCT and two using laboratory pathway) were commissioned to identify and invite up to 450 eligible patients who have not yet received an initial NHS Health Check invite letter. Practices were asked to record response rates to invite letter and rates of subsequent attendance or missed appointments to facilitate calculation of actual DNR and DNA rates for use in mathematical model using microcosting approach.

Although all four practices identified eligible patients and sent out invite letters, only two practices using POCT reported their findings. Recording response rates was very time consuming for practices using the laboratory pathway. These practices cited 'conflicting priorities', 'resources issues' and 'practicalities' in completing this specific component of the study. The number of patients who did not respond (DNR) to the initial invite letter was recorded by two GPs using POCT. The two GPs using POCT invited a total of 826 eligible patients, of which 75 patients completed an NHS Health Check within 4 months of initial invite. The average DNR rate following initial mail of either batch of invite letters from POCT practices was $90.2 \%$. Although data on patients responding to invites from laboratory practices were not available, the DNR rate is expected to be similar for all practices (regardless if they use POCT or not) in the same area with similar demography attempting to engage with the same representative sample population. In view of this, DNR rates for both POCT and non-POCT practices were assumed to be the same, and an average value for DNR of $90.2 \%$ was used in the analysis.

The average percentage of patients who responded to the invite letter and proceeded to book an appointment but ultimately DNA their NHS Health Check appointment in POCT practices was $10.6 \%$. For the subsequent steps where DNA is possible with laboratory pathway (ie, phlebotomy appointment, scheduled Health Check appointment for baseline data collection and second visit to learn about CVD risk score), a DNA rate of $5 \%$ at each bifurcation in the process tree was assumed. The choice of the 5\% DNA has been made using the national data of DNA rate of $7.5 \%$ as a reference. ${ }^{2}$ Decreasing the DNA rate to 5\% (as opposed to $7.5 \%$ from national data) for the laboratory pathway in subsequent bifurcations was to mitigate the risk of penalising the laboratory test attendance in favour of the POCT pathway, which has no opportunity for subsequent DNA after the initial NHS Health Check appointment. Therefore, the analysis conducted was 'worst case' scenario for POCT pathway.

\section{Mathematical model using microcosting approach}

A microcosting analysis was undertaken from the NHS perspective. Costs were calculated using 2013-2014 rates expressed in Great British Pound. As the analysis was performed assuming a time horizon of less than 1 year, the discounting of costs was deemed unnecessary.

The analysis used a decision tree to describe the options being compared and the possible pathways following them. This decision tree was based on the simplified flow diagram (figure 1) describing the observed process elements leading to an NHS Health Check in either laboratory $(\mathrm{LAB})$ or POCT pathway.

Costs were assessed using a microcosting approach. For each phase of the pathway, all resources (ie, staff, materials and consumables) were identified using data from laboratory engagement, GP engagement and observational data. Staff payments were estimated using the data on the average cost per hour in the UK from the Personal Social Service Research Unit 2014. The average cost per hour was multiplied by the time dedicated to each activity. The costs of the materials and consumables were assessed using data from the manufacturers, GPs, laboratory and evidence from the literature.

Because GPs do not routinely conduct an NHS Health Check during a consultation with patients, modelling was based on healthcare assistant (HCA) or nurse salary costs. The modelling was based on a commonly observed base case scenario: HCA-led Health Check with a DNR rate of $90.2 \%$ in both pathways, a DNA rate of $10.6 \%$ in the POCT and the first step of laboratory pathway and then a DNA rate of $5 \%$ in the following steps for the laboratory pathway; LAB fasting glucose measurement as opposed to HbAlc. The cost of the POC device (range $=£ 950-£ 1500$ ) and the costs of staff training on use of POCT were not used in the base case. The flowchart and decision tree are depicted in figure 1 . The probabilities associated with each event were obtained from real-world estimations from two POCT practices recruited into this project.

\section{SENSITIVITY ANALYSIS}

A one-way sensitivity analysis was undertaken, varying all input parameters to the original Base Case Scenario between their lower and upper values (table 1) to see how this would change the difference in total costs per 100 invites and to express the results in a tornado diagram. We included cost of device (range $=£ 950-£ 1500$ divided by 2000 tests) and training costs (8hours of HCA time divided by 2000 tests) in the sensitivity analysis.

A probabilistic sensitivity analysis was performed to obtain $95 \%$ credible intervals around the main results. This was done by carrying out 1000 simulations using uniform distributions for each parameter.

\section{ALTERNATIVE SCENARIOS}

Microcosting analysis and modelling resulted in the identification of comparative cost of NHS Health Check in 


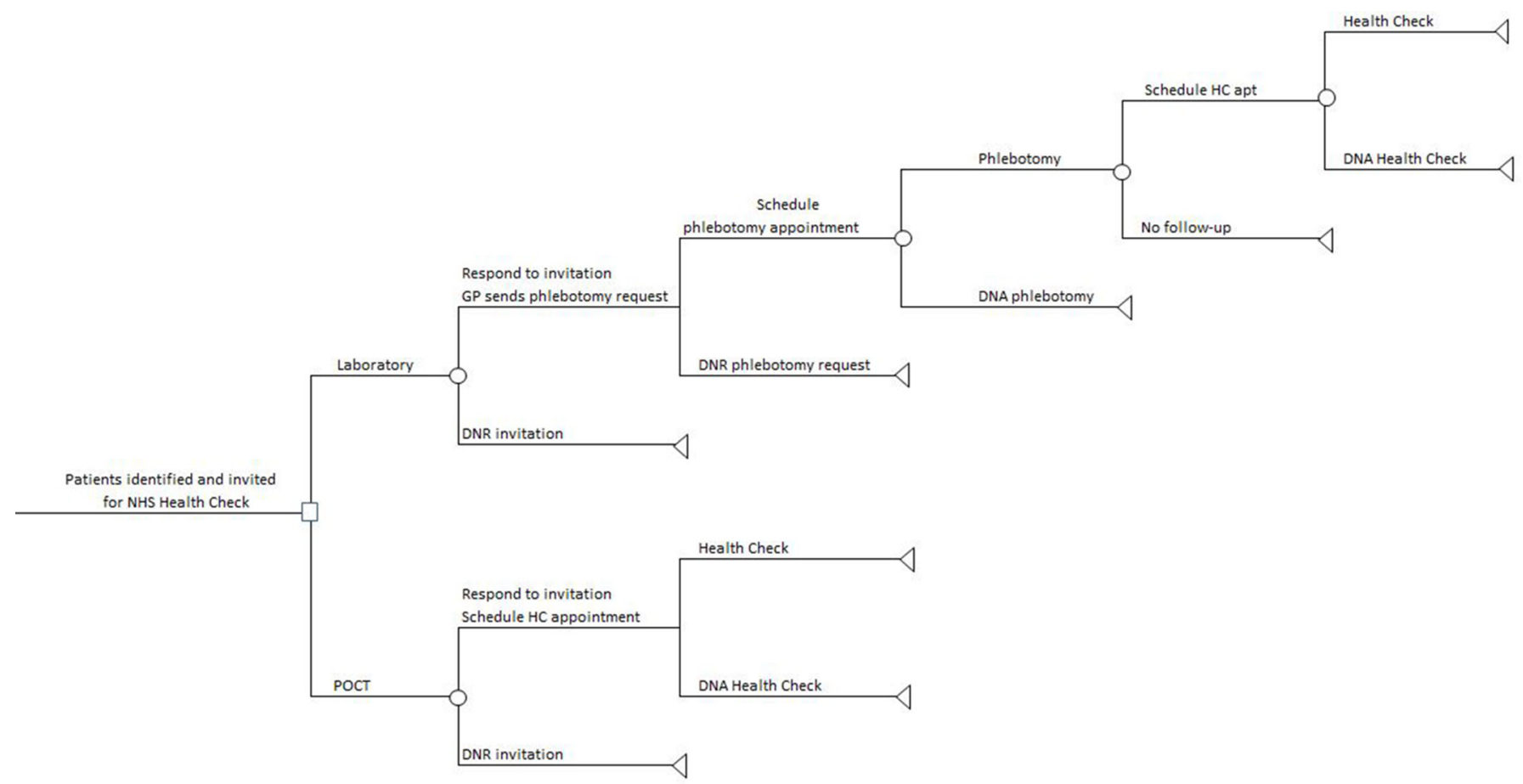

Figure 1 Decision tree showing laboratory (model 1) and POCT (model 2) NHS Health Check driven pathway. DNA, do not attend; DNR, do not spend; GP, general practice; HC, Health Check; POCT, point-of-care testing; NHS, National Health Service.

either Laboratory or POCT-driven practices to illustrate differences in the cost of one completed NHS Health Check in either pathway. We also performed the analysis for alternative scenarios: (1) cost of NHS Health Check assuming all patients respond to invite letter and complete the Health Check and (2) cost of an 'opportunistic' NHS Health Check as described below.

\section{COST OF AN OPPORTUNISTIC NHS HEALTH CHECK}

In some cases, it is theoretically possible that a patient may be offered the opportunity to complete a Health Check 'on the spot' after being identified as eligible following routine consultation or visit to general practitioners. This is less likely in the laboratory pathway as most patients will probably need to attend phlebotomy appointment first (laboratory-1 pathway). In case of availability of a recent ( $<3$ months) blood test indicating glucose and/ or HbAlc and total cholesterol levels on the patient's electronic health record (EHR), it would be possible for the patient to complete an 'opportunistic' Health Check (laboratory-2 pathway) pending availability of nurse or HCA. Because no information on the number of patients that would be offered an opportunistic Health Check or how many patients would DNA follow-up appointments in laboratory pathway was available, the total expected cost (TEC) of these options could not be calculated in the main model. However, the costs for one patient who completes an opportunistic Health Check for either pathway could be calculated.

\section{Ethics}

Ethical approval was not sought for this study as it did not involve recruitment of participants or use of patient identifiable data and other qualifying criteria. This was confirmed by completing the Health Research Authority (HRA) online decision tool for ethics, as evidenced by the HRA Tool Output Page.

\section{RESULTS}

Understanding patient flow and process times to administer an NHS Health Check

Patients undertaking an NHS Health Check in the laboratory pathway have two or three opportunities to miss an appointment or exit the care pathway, whereas patients undertaking an NHS Health Check in the POCT pathway only had one opportunity to miss the initial appointment (figure 1).

The time required to conduct an NHS Health Check up to the point of CVD risk score calculation varied from practice to practice (range $=20-40 \mathrm{~min}$ ) irrespective of whether they were using POCT. The shortest time recorded was for a simulated patient undertaking an opportunistic Health Check assuming that recent ( $<3$ month) blood results, and blood pressure readings were available on the patient's EHR.

The average time required to acquire a blood sample was $2.5 \mathrm{~min}$ longer with the cannulated syringe method used in the laboratory pathway than via the finger pin prick sampling method used in the POCT pathway. Events related to drawing a blood sample with a cannulated syringe include rolling up the patient's sleeve, applying 
a constriction band, labelling the blood vials, disinfecting the injection site, inserting the cannula, attaching the collection vials, drawing of blood sample, removing the cannula, applying digital pressure followed by a plaster over the wound and preparing the vials for dispatch. Accessing a blood sample with the finger prick method involved fewer steps, including disinfecting the injection site, finger pin prick, transferring blood aliquot onto a cassette via capillary tube to enable measurement with POC device and applying a plaster to the wound area. The 5 min time required for the POC device to deliver the test results was routinely used to apply the sphygmomanometer enabling further interlocution with the patient to drive the Health Check.

Adherence of GPs to PHE quality care criteria for using POCT All seven GPs using POCT (100\%) demonstrated that their staff were (1) suitably trained to use POC device, (2) that an appropriate internal quality control process was in place as evidenced by daily control sample and monthly calibration of the POCT device and (3) that each POCT location was registered in an accredited External Quality Assessment Programme. Only five (71\%) of seven practices confirmed that they had a nominated POCT coordinator. The overall score for compliance to the four PHE Quality Care Criteria for Using POCT was $92 \%$ for all seven practices.

\section{Results of the model}

Without taking into account DNR and DNA rates, one entire Health Check in the laboratory pathway would cost $£ 22.32$ ( $£ 3.28$ for the response to the letter and sending a phlebotomy request, $£ 1.02$ for scheduling a phlebotomy appointment, £10.33 for phlebotomy and blood tests, $£ 1.02$ for scheduling the Health Check appointment and $£ 6.67$ for the final Health Check appointment). One entire Health Check in the POCT pathway would cost $£ 17.04$ ( $£ 1.02$ for the response to the letter and scheduling the Health Check appointment and £16.02 for the Health Check appointment including POCT blood tests). Therefore, without taking into account DNR and DNA, one completed Health Check using POCT would cost $£ 5.28$ less than one completed using laboratory pathway.

Because of the higher number of visits needed for the laboratory pathway, there is a higher risk of subsequent DNA for the entire pathway compared with POCT pathway, which delivers the Health Check in one sitting. The calculated number of completed Health Checks per 100 invites and the costs per one completed Health Check are shown in table 2. The TEC of NHS Health Check per 100 patients in the base case scenario was £29 lower (95\% credible interval=-£244 to £95) in POCT-led pathway than in laboratory pathway. The TEC per one completed Health Check was $£ 7$ lower in the POCT pathway $(\mathrm{TEC}=18)$ than in the laboratory pathway $(\mathrm{TEC}=£ 25)$ when DNA and DNR rates are taken into account.

\section{One-way sensitivity analysis}

The value of each parameter was varied between the lower and the upper values to check how this would influence 
Table 2 Base case scenario showing total expected cost (TEC, number of completed Health Checks and costs per completed Health Check per 100 invites (including 95\% credible intervals from probabilistic sensitivity analysis (PSA))

\begin{tabular}{llll}
\hline Model & TEC (range) & $\begin{array}{l}\text { No. of NHS Health Check } \\
\text { completed (range) }\end{array}$ & $\begin{array}{l}\text { TEC per completed NHS Health } \\
\text { Check (range) }\end{array}$ \\
\hline LAB-led & $£ 189.00(£ 164$ to $£ 561)$ & $7.50(5.0$ to 10.7$)$ & $£ 25(£ 25$ to $£ 51)$ \\
POCT-led & $£ 160.00(£ 117$ to $£ 354)$ & $8.74(5.8$ to 11.9$)$ & $£ 18(£ 17$ to $£ 36)$ \\
Difference & $-£ 29(-£ 244$ to $£ 95)$ & $1.25(-3.6$ to 5.5) & -£7 (-£23 to -£2) \\
\hline
\end{tabular}

NHS, National Health Service; POCT, point-of-care testing; TEC, total expected cost.

the difference in TECs per 100 invites. The tornado diagram (figure 2) shows the 10 parameters with the largest influence on the results. Only three parameters had a large enough impact on the results to change the difference from negative to positive rendering the POCT model more expensive in this case.

When the response to the invitation in laboratory pathway was low or the response to the invitation in POCT pathway was high, total costs per 100 invites were higher for POCT. In this case, the number of completed Health Checks in the POCT pathway was higher, and the costs per completed Health Check were equal to the base case.

When the HCA or nurse time for the Health Check was high, the total costs per 100 invites were higher for POCT than for the LAB model, the number of completed Health Checks was the same as in the base case and the cost per completed Health Check for POCT was higher than that in the base case (up to £22) but still lower than that in the LAB model.

\section{Alternative scenarios}

\section{Cost of Health Check assuming all patients respond to invite} letter and complete the Health Check

If we assume all invited persons would respond in both the laboratory pathway and the POCT pathway, but some people would drop out of the pathway because of missed appointments (ie, DNR rate is $0 \%$, but DNA rate as in the base case model), 77 Health Checks would be completed in the laboratory pathway compared with 89 in the POCT pathway. The TEC per one completed Health Check would be the same as when taking into account observed

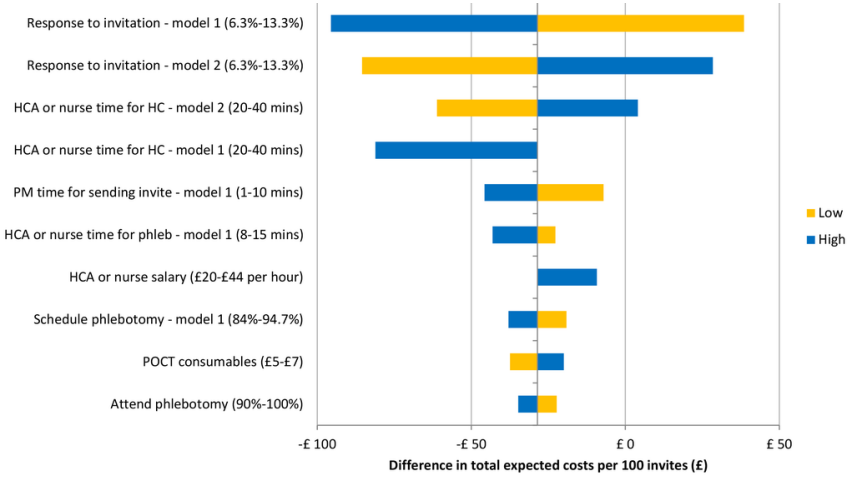

Figure 2 Tornado diagram showing the 10 parameters with the largest influence on the difference in costs per 100 invites. $\mathrm{HC}$, health care; HCA, healthcare assistant; POCT, point-of-care testing.
DNR rates $(\mathrm{TEC}=£ 25$ for Laboratory and $£ 18$ for POCT; table 3).

\section{Cost of opportunistic Health Checks}

The costs for one patient who completes an opportunistic NHS Health Check for either pathway are shown in table 4. The costs for an opportunistic health check via laboratory-1 pathway (ie, phlebotomy at GP) would be $£ 21.67$, which is effectively the costs of the normal laboratory pathway minus the costs of sending a letter with the phlebotomy request (£0.65/patient). An opportunistic Health Check in laboratory-2 pathway (ie, no phlebotomy indicated as recent blood results are available on patient's EHR) would cost £7.69 less. An opportunistic Health Check using POCT would cost the same as the normal POCT pathway (£17.04). For all opportunistic Health Checks, the costs of sending an invitation letter (calculated at £21.93 per 100 invites, but not included in the main model because it applies to all pathways) can be omitted.

\section{Discussion}

This is the first study using a cost-minimisation analysis to investigate the potential cost-saving use of POCT in delivering NHS Health Check in the primary care setting. According to the results of the mathematical model, the cost savings to the NHS associated with POCT were estimated at £29 per 100 patients.

The total excepted cost of a POCT-driven NHS Health Check in GP was likely to be lower than the traditional laboratory pathway up to the point of CVD risk score presentation. This applied to all scenarios, including those scenarios that factored in the cost of the device and the cost of staff training on use of POCT, both when looking at the cost of a Health Check for 100 patients, and the per-patient cost for one completed Health Check. The only exception to this was when a patient was offered an opportunistic Health Check following a routine consultation visit to the GP where the patient's EHR indicated recent ( $<3$ months) blood results for glucose and/ or HbAlc and cholesterol levels (£7.69 for laboratory vs $£ 17.04$ for POCT). A 4-year study found $25 \%$ of Health Checks completed in GP to result from opportunistic recruitment with $75 \%$ of all Health Checks completed using prescheduled appointments. ${ }^{11}$ It is unknown what proportion of all NHS Health Check are delivered using POC testing. 
Table 3 Alternative scenario showing total expected cost (TEC costs, number of completed health checks and costs per completed health check per 100 invites assuming all patients respond to invite letter

\begin{tabular}{llll}
\hline & \multicolumn{2}{c}{ Alternative scenario assuming all patients respond to invite letter } \\
\cline { 2 - 4 } Model & Total Expected Costs (TEC) & $\begin{array}{l}\text { No of NHS Health Check } \\
\text { completed }\end{array}$ & $\begin{array}{l}\text { TEC per completed NHS Health } \\
\text { Check }\end{array}$ \\
\hline LAB-led & $£ 1932$ & 76.62 & $£ 25$ \\
POCT-led & $£ 1640$ & 89.37 & $£ 18$ \\
Difference & $-£ 292$ & 12.75 & $-£ 7$ \\
\hline
\end{tabular}

NHS, National Health Service; POCT, point-of-care testing; TEC, total expected cost.

There is wide variation in how the NHS Health Check Programme is currently being delivered. ${ }^{11}$ This study illustrated a number of possible entry points that would enable a patient to undertake an NHS Health Check in GP (figure 1). However, in contradistinction to the POCT pathway which delivers the Health Check in one sitting, the number of bifurcations in the laboratory pathway imply that patients have three times more opportunities to miss a health check-related appointment. Missed appointments represent a significant burden on NHS resources, ${ }^{12}$ with up to $9 \%$ of total outpatient appointments missed in $2014-15^{13}$. While it is difficult to establish the exact financial impact of missed GP appointments, missed first outpatient appointments were estimated to cost the NHS up to $£ 225$ million in 2012-13 ${ }^{14}$. Use of POCT to deliver NHS Health Check in one sitting could result in fewer missed GP or outpatient/phlebotomy appointments with cost savings from the NHS perspective.

\section{Cost minimisation using POCT for routine NHS Health Check}

There is much interest in reducing costs and in improving overall effectiveness of CVD screening, and the NHS Health Check Programme is the first such national CVD risk assessment and management programme in England. The Department of Health's modelling of the programme's cost-effectiveness was based on the assumption that the NHS Health Check programme would be widely implemented with good overall uptake. ${ }^{6}$ However, a 4-year evaluation of the NHS Health Check programme reported low coverage $(21.4 \%)$ suggesting that there is significant room for improvement in delivering the programme more effectively. ${ }^{15}$ A wide variation in coverage between English regions $(9.4 \%$ to $30.7 \%)$ and between GPs ( $0 \%$ to $72.7 \%$ ) and among ethnic minorities suggests that the programme is not delivered equitably throughout England. ${ }^{8}$ By lowering the opportunity for missed appointments, POCT may offer an effective way to increase coverage while also reducing costs associated with missed appointments.

A recent evaluation of New Zealand's Heart Check Programme (NZHCP) showed that whereas the coverage rate was initially poor, the programme eventually met its national target coverage rate of $90 \%$ following a series of patient engagement initiatives coupled to effective use of IT. ${ }^{16}$ The evaluation identified the need for blood tests as an impediment to slow uptake initially, and case studies involving the use of POCT to support NZHCP were deemed effective. However, use of POCT in NZHCP was considered too expensive from the perspective of the provider, although it is unknown whether this conclusion resulted from a cost-minimisation analysis. ${ }^{16}$

In the present analysis, we assumed that a Health Check would require a minimum time of $20 \mathrm{~min}$ (lower limit) and up to $40 \mathrm{~min}$ (upper limit). The mathematical model assumed a base case of $20 \mathrm{~min}$ for the laboratory pathway and $30 \mathrm{~min}$ for the POCT pathway. The discrepancy, which was in favour of the laboratory pathway, was included because we acknowledged that not all patients in the laboratory pathway will have a blood test at the GP (i.e, some patients will have phlebotomy in pathology services laboratory). We also assumed 5\% DNA rate for

\begin{tabular}{llll}
\hline \multicolumn{2}{l}{ Table 4} & Costs for one patient completing an opportunistic & NHS Health Check at GP in either pathway \\
\hline Event & $\begin{array}{l}\text { Laboratory-1 } \\
\text { (phlebotomy) }\end{array}$ & $\begin{array}{l}\text { Laboratory-2 } \\
\text { (no phlebotomy at GP) }\end{array}$ & $\begin{array}{l}\text { POCT } \\
\text { (via pin prick sample) }\end{array}$ \\
\hline $\begin{array}{l}\text { GP hands out a phlebotomy } \\
\text { request }\end{array}$ & $£ 2.63$ & - & - \\
$\begin{array}{l}\text { Schedule phlebotomy } \\
\text { appointment }\end{array}$ & $£ 1.02$ & - & - \\
Phlebotomy & $£ 10.33$ & - & - \\
Schedule health check & $£ 1.02$ & $£ 1.02$ & $£ 1.02$ \\
$\begin{array}{l}\text { CVD risk score presentation } \\
\text { Total cost }\end{array}$ & $£ 6.67$ & $£ 6.67$ & $£ 16.02$ \\
\hline
\end{tabular}

CVD, cardiovascular disease; GP, general practitioner; NHS, National Health Service; POCT, point-of-care testing. 
subsequent Health Check-related appointments in the laboratory pathway as opposed to $7.9 \%$ national average rate in order to not penalise this pathway. In spite of this, and considering that the costs of POC devices with similar capabilities are likely to decrease as technology and related markets develop further, the results of this cost-minimisation analysis, which represent a 'worst case' scenario for POCT pathway, are still in favour of using POC to support NHS Health Check in the primary care setting from the NHS perspective.

\section{Extant barriers for widespread adoption of POCT to drive NHS Health Check}

The findings of this study illustrated routine use of POCT in GP to support NHS Health Check in the contemporary setting, with good overall compliance of the GPs to the four PHE Care Quality Criteria for using POCT. ${ }^{10}$ However, there are a number of extant barriers to the widespread adoption and diffusion of POCT in primary care generally, ${ }^{17-19}$ including current reimbursement models and misaligned incentives. ${ }^{17}$ Whereas other barriers such as prevailing attitudes of healthcare practitioner or a preference to use laboratory services over POCT can be overcome with relatively minor changes to work pattern, structural barriers including the lack of a suitable funding and reimbursement model will require policy change if widespread NHS adoption is to be achieved. ${ }^{1720}$ Evidence of cost savings to the NHS from using POCT may provide a rationale for a change in the current reimbursement model and incentives to GPs in favour of wider adoption and use of POCT for specified purposes. POC device manufacturers could also offer a discounted pricing policy for test panels to increase their market presence and to overcome the perceived test cost procurement barrier when introducing POCT more extensively within the NHS for specific purposes. ${ }^{21}$ The recently proposed changes to the NHS Pathology tariff are likely to positively affect the general adoption and diffusion of POCT in the UK. However, further research is needed to develop scaling-up strategies to address the resourcing, clinical governance and economic impact of widespread NHS implementation for the purpose of supporting NHS Health Check in the primary care settingP.

\section{Implications of the study}

By facilitating screening in a single visit while offering satisfactory accuracy of testing, POCT has clear advantages over the laboratory-based approaches, which may be time consuming. ${ }^{22}$ POCT also carries the potential of improved patient care, with lower recurrent hospital admissions and economic savings for preventable chronic conditions..$^{20}$ These patient benefits may occur in the hospital setting, in GP, in the community setting and in remote areas. ${ }^{62324}$ There is growing interest internationally and in the UK among clinicians to use POC testing to help diagnose a range of acute and chronic conditions ${ }^{25}$ and in supporting antibiotic stewardship. ${ }^{17} 26-28$ However, unless the cost of the tests (including equipment, consumables and time) were fully reimbursed, the practice could lose money from using POCT. ${ }^{17}$ This is one of the factors that has hindered the uptake of POCT in the primary care setting in England.

This study shows that the NHS Health Check programme would cost less from the NHS perspective if POCT was used at scale. Future large-scale public health screening programmes should ideally consider a disruptive commissioning model that includes reimbursement and other incentives to affect the large-scale adoption of suitable multi-array POC devices in GP with the potential to reduce costs from the NHS perspective.

Low statin prescribing to people at increased CVD risk and variation in statin prescribing among GPs also highlights the need for better follow-up of patients. ${ }^{89} 29$ Because CDV risk score is available immediately following a Health Check with POCT, it could potentially expedite the timely prescription of statins to patients identified to be at high CVD risk during a routine or follow-up appointment with GP or prescribing clinician.

Advances in technology and miniaturisation could result in more affordable POC devices with added functionality to support different clinic needs in routine general practice. Competitive behaviour between POC device manufacturers could lead to lower costs and increased quality of base unit and consumables, making POC more affordable from the NHS perspective. Use of POCT to support delivery of NHS Health Check in one sitting may also be more convenient from the patient's perspective as this diminishes the need for subsequent visits and may be preferred by patients who are needle-phobic and avert to having a blood test using the cannulated syringe method. ${ }^{9}$

\section{Limitations of study}

The principle limitation of this study was the lack of availability of data for subsequent DNA rates in the laboratory-led pathway. However, an evidence-based assumption was used to for subsequent DNA rates with a fixed value of $5 \%$, which is significantly lower than the observed rate of DNA in POCT pathway and lower than the national average rate of missed appointments. The results of this analysis are conservative in showing the savings associated with POCT, which may be higher if subsequent DNA rates are above 5\%, as is likely to be the case. Further, whereas this study employed the use of a mathematical model with a microcosting approach, future studies could determine if use of POCT to deliver NHS Health Check is cost-effective from the NHS perspective by taking into account other variables and outcome measures post CVD risk score presentation.

\section{Conclusion}

A cost-minimisation analysis using a mathematical model with alternative scenarios showed that the total expected cost of using POCT to deliver a routine NHS Health Check in the primary care setting up to the point of CVD risk score presentation is lower than the laboratory-led pathway from the NHS perspective. Laboratory-led pathway offers patients three times more opportunities 
to miss subsequent NHS Health Check-related appointments or to exit the care pathway compared with POCT pathway. Using POCT in this context could be more convenient for patients and offers GPs the ability to act as a 'one stop shop' to patients by delivering a complete NHS Health Check in a single sitting. Using POCT in routine general practice for this purpose will likely reduce overall programme costs while supporting an increase in coverage from existing levels.

Contributors All authors provided substantial contributions to the conception (AEO, AM, GH, JH, ZN and MS), design (AEO, MS, MW, GH, JH and ZN), acquisition of the data (AEO and CD) or analysis and interpretation of the study data (AEO, CD and $M W$ ) and approved the final version of the paper. AEO took the lead in planning the study with support from the co-authors and carried out the data analysis with support from CD. AEO also wrote the first draft of the paper, which was then revised by all co-authors. The design, development and analysis of the model, including the sensitivity analyses, were carried out by EP and TV. AEO is the guarantor.

Funding Unconditional funding for this work was provided by Alere International Limited. The Funder did not have a role in study design or analysis. The research was supported by the NIHR Diagnostic Evidence Co-operative (DEC) London. EP was supported by the National Institute for Health Research (NIHR) Collaboration for Leadership in Applied Health Research and Care (CLAHRC) North Thames at Bart's Health NHS Trust. The views expressed are those of the authors and not necessarily those of the funder, the NHS, the NIHR or the Department of Health. The Department of Primary Care \& Public Health at Imperial College London is grateful for support from the NW London NIHR Collaboration for Leadership in Applied Health Research \& Care (CLAHRC) and the Imperial NIHR Biomedical Research Centre.

\section{Competing interests None declared.}

Patient consent Detail has been removed from this case description/these case descriptions to ensure anonymity. The editors and reviewers have seen the detailed information available and are satisfied that the information backs up the case the authors are making.

Provenance and peer review Not commissioned; externally peer reviewed. Data sharing statement № additional data are available.

Open Access This is an Open Access article distributed in accordance with the Creative Commons Attribution Non Commercial (CC BY-NC 4.0) license, which permits others to distribute, remix, adapt, build upon this work non-commercially, and license their derivative works on different terms, provided the original work is properly cited and the use is non-commercial. See: http://creativecommons.org/ licenses/by-nc/4.0/

(c) Article author(s) (or their employer(s) unless otherwise stated in the text of the article) 2017. All rights reserved. No commercial use is permitted unless otherwise expressly granted.

\section{REFERENCES}

1. World Health Organisation2007Prevention of cardiovascular disease-guidelines forassessment and management of cardiovascular risk

2. Murray CJ, Richards MA, Newton JN, et al. UK health performance: findings of the Global Burden of Disease Study 2010. Lancet 2013;381:997-1020.

3. Department of Health. Putting prevention first- vascular checks: risk assessment and management - next steps guidance for primary care trusts. 2009, 2009. http://www.dh.gov.uk/en/Publicationsandsta
tistics/Publications/PublicationsPolicyAndGuidance/DH_090277. (accessed 7 July 2016).

4. England PH. NHS Health check best practice guidance, 2015.

5. England PH. NHS Health check: our approach to the evidence, 2013.

6. Department of Health. Vascular checks: economic Modelling for vascular checks. 2008, 2008. http://www.dh.gov.uk/en/Publicatio nsandstatistics/Publications/PublicationsPolicyAndGuidance/DH_ 085869. (accessed 12 June 2015).

7. Chang KC, Lee JT, Vamos EP, et al. Impact of the National Health Service Health Check on cardiovascular disease risk: a difference-indifferences matching analysis. CMAJ 2016;188:E228-E238.

8. Chang K, Millett $\mathrm{C}$, Soljak M, et al. National coverage of the English NHS Health Check programme. Eur J Public Health 2014;24.

9. Robson J, Dostal I, Sheikh A, et al. The NHS Health Check in England: an evaluation of the first 4 years. BMJ Open 2016;6:e008840.

10. England PH. NHS Health check programme standards: a framework for quality improvement: PHE publications gateway number: 2013503 ed, 2014.

11 Nicholas JM, Burgess C, Dodhia H, et al. Variations in the organization and delivery of the 'NHS health check' in primary care. $J$ Public Health 2013;35:85-91.

12. NHS Digital.. Hospital outpatients: appointments top 100 million for first time in 2013-14, 2015.

13. Department of Health. A zero cost way to reduce missed hospital appointments. Crown Copyright 2016.

14. Office NA. NHS waiting times for elective care in England, 2014.

15. Robson J, Dostal I, Sheikh A, et al. The NHS Health Check in England: an evaluation of the first 4 years. BMJ Open 2016;6:e008840.

16. Allen and Clarke MoHNZC. More Heart and Diabetes Checks evaluation: final Report. Minisry of Health New Zealand 2016:35-6.

17. Huddy JR, Ni MZ, Barlow J, et al. Point-of-care $\mathrm{C}$ reactive protein for the diagnosis of lower respiratory tract infection in NHS primary care: a qualitative study of barriers and facilitators to adoption. BMJ Open 2016;6:e009959.

18. Shaw JLV. Practical challenges related to point of care testing. Pract Lab Med 2016;4:22-9.

19. Larsson A, Greig-Pylypczuk R, Huisman A. The state of point-of-care testing: a European perspective. Ups J Med Sci 2015;120:1-10.

20. Jones $\mathrm{CH}$, Howick J, Roberts NW, et al. Primary care clinicians' attitudes towards point-of-care blood testing: a systematic review of qualitative studies. BMC Fam Pract 2013;14:117.

21. FitzGibbon F, Huckle D, Meenan BJ, et al. Barriers affecting the adoption of Point-of-Care Technologies used in chest pain diagnosis within the UK National Health Service: part 2-Manufacturer pricing and reimbursement policy issues. Point of Care 2010;9.

22. Whitehead SJ, Ford C, Gama R. The impact of different point-of-care testing lipid analysers on cardiovascular disease risk assessment. $J$ Clin Pathol 2014;67:535-9.

23. Schilling UM. The economic benefits of point-of-care testing, 2015.

24. Hortin GL. Does Point-Of-Care Testing save money or cost more? Labmedicine 2005.

25. Howick J, Cals JW, Jones C, et al. Current and future use of pointof-care tests in primary care: an international survey in Australia, Belgium, The Netherlands, the UK and the USA. BMJ Open 2014;4:e005611.

26. Cals JW, Ament AJ, Hood K, et al. C-reactive protein point of care testing and physician communication skills training for lower respiratory tract infections in general practice: economic evaluation of a cluster randomized trial. J Eval Clin Pract 2011:17:1059-69.

27. Jakobsen KA, Melbye H, Kelly MJ, et al. Influence of CRP testing and clinical findings on antibiotic prescribing in adults presenting with acute cough in primary care. Scand J Prim Health Care 2010;28:229-36.

28. Van den Bruel A, Jones C, Thompson M, et al. C-reactive protein point-of-care testing in acutely ill children: a mixed methods study in primary care. Arch Dis Child 2016;101:382-6.

29. Artac M, Dalton AR, Majeed A, et al. Uptake of the NHS Health Check programme in an urban setting. Fam Pract 2013;30:426-35. 